\title{
The transitional plasticity of ammonium chloride
}

\author{
F. J. Bartis \\ Department of Physics, Indiana University, Bloomington, Indiana 47401, U.S.A.
}

(Reçu le 21 mars 1979, révisé le 28 mai 1979, accepté le 29 mai 1979)

\begin{abstract}
Résumé. - D’une théorie phénoménologique de la cinétique des domaines dans une zone diphasée cohérente on déduit que la vitesse de fluage au début de la déformation macroplastique s'annule lorsque la température s'approche du point singulier du temps de relaxation thermique. On signale que l'érosion des domaines qui accompagnent les dislocations coins peut être observée à l'aide de l'intensité de la lumière diffusée à 90 degrés.
\end{abstract}

\begin{abstract}
From a phenomenological theory of domain kinetics in a coherent two-phase field it is inferred that the creep rate at the start of macroplastic deformation goes to zero, as the temperature approaches the singular point of the thermal relaxation time. It is pointed out that the erosion of the domains decorating the edge dislocations can be followed through the intensity of the light scattered at 90 degrees.
\end{abstract}

The Cottrell theory of impurity atmosphere drag is remarkably successful in accounting for the microcreep of tin and the repeated yielding of iron near room temperature [1, 2]. It is taken here as a model for the construction of a theory of coherent precipitate drag. I first assess the force of attraction between an edge dislocation and a nearby precipitate. Then I evaluate the strain rate at the onset of macroplastic deformation under the assumption that a slowly moving dislocation gains its freedom when the precipitate, trying to keep up with the dislocation, becomes noticeably stunded. Lastly I propose a study of the transitional opalescence of a crystal under a small external stress to determine how a typical precipitate fares during the passage from microplasticity to macroplasticity.

The mechanical effects of a precipitate with a different composition are extensively discussed in the literature. On the other hand, deformation when the precipitate has the same composition as the matrix receives scant attention. To correct this imbalance I want to consider ammonium chloride during the discontinuous stage of its orientational ordering reaction. Because the transition line of the reaction has a positive slope, the crystallites on the constricted side of an edge dislocation pass from the disordered to the partially ordered form of the material at higher temperatures. As long as the ambient temperature exceeds the nominal ordering point at atmospheric pressure, a roughly cylindrical domain of the partially ordered modification appears alongside the dislocation. This domain can be expected to contribute to the elastic scattering of light, for its refractive index is different from that of the disordered matrix. It should also add to the elastic deformation of the material, as it tends to lock the dislocation at its side.

According to Cottrell and Jaswon [1] a tin crystal exhibits microplasticity, when the average edge dislocation has to drag along an impurity atmosphere. By analogy, I expect ammonium chloride to manifest a slow creep in the coexistence region of the orientational transition, when a typical edge dislocation must drag along a domain of the minority phase. Probably the simplest way to estimate the drag is through the change in the elastic strain energy, when the domain is displaced a minuscule distance $s$ relative to the dislocation. As a first approximation, the stress around an isolated dislocation can be taken to be

$$
P \approx G b / 2 \pi R,
$$

where $G$ denotes the shear modulus and $b$ is the length of the Burgers vector of the dislocation. It is easy to see in figure 1 that the change in the elastic strain energy comes in effect from the transformation of the material in the two crescent-shaped regions. Neglecting trigonometric factors, I obtain for the change in the elastic energy per unit length of the dislocation

$$
W \approx G b f s^{2} / 2 \pi R_{\mathrm{n}},
$$




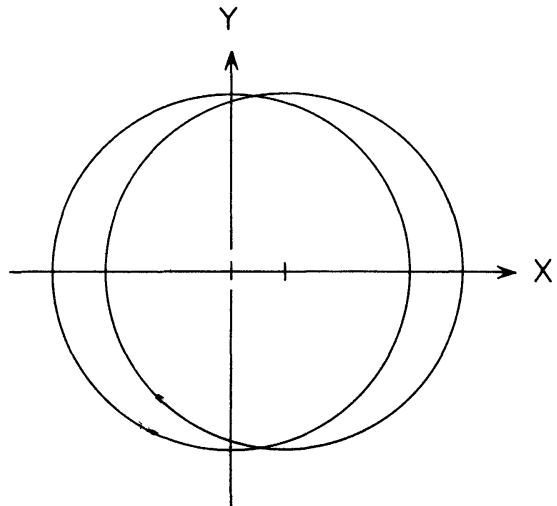

Fig. 1. - Diagram for the calculation of the attraction between an edge dislocation at the origin and a domain of the minority phase. The domain is shown in cross section at its equilibrium position and at a distance $s$ from this position.

where $R_{\mathrm{n}}$ is the radius of the domain and $f$ is the fractional change of volume in going from one phase to the other. Differentiation with respect to $s$ gives the drag

$$
D \approx G b f s / \pi R_{\mathrm{n}} .
$$

When the speed of the dislocation is very low, the domain radius differs little from its value at thermodynamic equilibrium. But as the speed increases, the domain can be expected to shrink a little. If $\tau$ is the relaxation time of the interphase boundary, then the domain should become noticeably stunted when it has to move a distance $2 R_{\mathrm{n}}$ in a time interval $\tau$. Let me assume that at the critical speed $2 R_{\mathrm{n}} / \tau$ the dislocation just outstrips the domain and the solid begins to yield. Inserting the expression for $\tau$ found in my earlier work on domain kinetics in simple order-disorder transitions [3], I get for the critical strain rate

$$
\mathrm{d} \varepsilon / \mathrm{d} t=2 \rho b C A / R_{\mathrm{n}} .
$$

Here $\rho$ is the dislocation density, $C$ is a material constant and $A$ is a factor of the Arrhenius form, i.e., $A=A_{0} \exp (-H / k T)$. Since the equilibrium radius goes as $\left|T-T_{0}\right|^{-1}$, the solid can be expected to deform plastically when the strain rate attains a value

$$
\mathrm{d} \varepsilon / \mathrm{d} t=M\left|T-T_{0}\right| \exp (-H / k T),
$$

where $M$ is a constant for a given sample, $H$ is an activation energy and $T_{0}$ is the singular point of the thermal relaxation time.

Eq. (5) can be given a simple physical interpretation. Domain growth is viewed as a two-step process. First some of the atoms change their positions within the lattice. Second the lattice alters its configuration. The initial step proceeds by thermal excitation over a potential barrier and so its speed is proportional to an Arrhenius factor. The second step, on the other hand, has its counterpart in the normal growth of a crystal from a solution or melt. The preponderance of the work on unassisted growth points to a simple proportionality between the speed and the undercooling of the crystal boundary. By analogy, the speed of the second step in domain growth is taken to be proportional to the effective undercooling of the boundary. Because the two steps are successive and independent, the speed of the interphase boundary is proportional to the product of the two elemental speeds. By assumption, a dislocation starts to break away from a domain when the interphase boundary can no longer maintain its position relative to the moving dislocation. Hence the critical strain rate is also proportional to the product of an Arrhenius factor and an effective undercooling. This is what eq. (5) says. It is interesting to note that, were the second step absent, the critical creep rate would be of the form

$$
\mathrm{d} \varepsilon / \mathrm{d} t=N \exp (-Q / k T),
$$

where $N$ is a constant for a given specimen and $Q$ represents an activation energy. As a matter of fact, the second step is missing in the formation of an impurity atmosphere. So eq. (6) is reasonable as the form of the strain rate at which metals of moderate purity start to yield [2].

It would be wrong to conclude from the foregoing discussion that the critical strain rate of tin near room temperature is higher than that of ammonium chloride during its orientational transition. The presence of an extra step in domain growth is not enough to decide the issue. For this purpose I need a lower bound on the critical creep rate of ammonium chloride. Near the transition point its thermal relaxation time is approximately 1 week [4]. In a crystal containing $10^{4}$ dislocations $/ \mathrm{cm}^{2}$ the domain radius should reach at least $10 \mu \mathrm{m}$. Thus I estimate the critical strain rate to be greater than $1.2 \times 10^{-12} / \mathrm{s}$. For purposes of comparison it may be recalled that carefully purified tin tends to deform plastically above a strain rate of $10^{-8} / \mathrm{s}$ [5]. Adopting the terminology of Chalmers [5], I view the critical strain rate as the dividing line between microplastic and macroplastic deformation. The macroplastic label certainly fits ammonium chloride near $242.9 \mathrm{~K}$. Lawson [6] reports that during his measurement of the isothermal Young's modulus the beam displacement increases steadily with time after the load is applied, at such a rate that the increase in 5 minutes is about half the initial displacement.

Justification for a special terminology can also be found in the question of the temperature range of plasticity. Lawson concludes from his observations of creep that ammonium chloride is plastic over a temperature interval of about $0.3 \mathrm{~K}$. Yet from a study of its mechanical behaviour at $36 \mathrm{kHz}$ he derives an internal friction curve with a full width of almost $1.7 \mathrm{~K}$ at half the peak height. One way to rationalize 
the different findings is suggested by eq. (5). It indicates that the temperature interval of macroplastic deformation depends on how large a microcreep rate the external stress produces. Ammonium chloride should exhibit macroplasticity over a greater range of temperatures when the stress is increased.

Crucial to the present theory is the presumption that the dislocations gain their release at the same time that the domains are rapidly decreasing in size. An experimental test of the simultaneity of these two events is certainly desirable. It may not be too difficult.
I suggest that light be scattered from an ammonium chloride crystal to which a small stress can be applied. If the domains of the minority phase shrink at the onset of macroplastic deformation, then the opalescence of the crystal should become weaker. Here I am assuming that the optical inhomogeneity of the crystal decreases, as the domains become smaller. To minimize the background level the observation should be made in the upper portion of the mixed phase region, where edge dislocations serve as the principal centres of domain growth $[7,8]$.

\section{References}

[1] Cottrell, A. H., JAswon, M. A., Proc. R. Soc. A 199 (1949) 104.

[2] Cottrell, A. H., Philos. Mag. 44 (1953) 829.

[3] Bartis, F. J., Phys. Lett. A 55 (1975) 311.

[4] Fredericks, G. E., Phys. Rev. A 4 (1971) 911.
[5] Chalmers, B., Proc. R. Soc. A 156 (1936) 427.

[6] Lawson, A. W., Phys. Rev. 57 (1940) 417.

[7] Bartis, F. J., Phys. Lett. A 43 (1973) 61.

[8] Pique, J. P., Dolino, G., Vallade, M., J. Physique 38 (1977) 1527. 